\title{
Eine Gründungsschrift der Technikwissenschaftsgeschichte in Deutschland
}

\section{Kommentar zu Gisela Buchheim: Zur Wechselwirkung von Naturwissenschaften und Technikwissenschaften in ihrer historischen Entwicklung (1978)}

\section{Thomas Hänseroth}

Dieser Artikel kann als Gründungsschrift des seit den späten 1970er Jahren im deutschsprachigen Raum entwickelten Forschungs- und Lehrprogramms einer Geschichte der Technikwissenschaften gelesen werden. Schauplatz war der Wissenschaftsbereich Geschichte der Produktivkräfte der Sektion Philosophie und Kulturwissenschaften an der Technischen Universität Dresden. Das an die Namen Gisela Buchheim (1931-1991) und Rolf Sonnemann (geb. 1928) geknüpfte kollektive Projekt folgte einem noch wenig beschrittenen Pfad. Buchheim, später zur außerordentlichen Professorin für Wissenschaftsgeschichte ernannt, war dessen Spiritus rector. Sonnemann, ordentlicher Professor für Wirtschaftsgeschichte und Geschichte der Produktivkräfte sowie Leiter des oben genannten Wissenschaftsbereichs, sorgte neben inhaltlichen Beiträgen vor allem mit Durchsetzungsvermögen und dank seiner Stellung als ein 
zentraler Akteur in den Netzwerken sowohl der Wissenschafts- als auch Technikhistoriker der DDR für die stabile Institutionalisierung in Form des 1978 an der TU Dresden gegründeten Zentrums für Geschichte der Technikwissenschaften (Buchheim/Sonnemann 1985, Hänseroth/Mauersberger 1996). An diesem Zentrum wurden gleichermaßen Forschung und Lehre betrieben, wofür zunächst mit zwei Asprirantenkursen (1978-1982 und 1983-1987), heute hieße dies Graduiertenkolleg, der Grundstock gelegt wurde. Da zu den Prämissen des Dresdener Konzepts zählte, dass aus den jeweiligen Fachwissenschaften kommende Akteure Technikwissenschaftsgeschichte schreiben sollten, dienten diese auch der auf ein Zweitstudium hinauslaufenden Vermittlung historischer Kompetenz (Buchheim/Sonnemann 1986).

Auffällig ist, dass sich der abgedruckte Aufsatz ausschließlich auf Publikationen aus der UdSSR bezieht und zudem in Teilen Rezensionscharakter besitzt. Gleichwohl folgte die Autorin weiterreichenden Intentionen. Der Bezug auf sowjetische Literatur besaß in den Gesellschaftswissenschaften der DDR bis zum Anbruch der Ära Gorbatschow auch die Funktion, gegen Kritik zu immunisieren. Dies war insofern für Buchheim geboten, als während der 1970er Jahre in der DDR umstritten war, ob der historischen Dimension der Produktivkraft Wissenschaft unter dem Dach der etablierten, konzeptionell technik-, wirtschafts- und arbeitsgeschichtliche Perspektiven verbindenden Geschichte der Produktivkräfte nachgespürt oder aber dies mit dezidiert wissenschaftshistorischen Ansätzen verfolgt werden sollte. Letzteres stieß auf starke Vorbehalte unter Produktivkrafthistorikern. Gleichermaßen kollidierte das von Buchheim verfochtene Konzept einer Geschichte der Technikwissenschaften mit dem weiter unten skizzierten Alleinvertretungsanspruch von Naturwissenschaftshistorikern. Sonnemann, der anfangs Buchheims Intentionen skeptisch gegenüber gestanden hatte, begriff bald die Chancen eines wissenschaftshistorischen Ansatzes. Geriet doch eine derartige Geschichte der Produktivkräfte, die letzten Endes auf der Basis der marxistischen Gesellschaftstheorie die Arbeit des viel zitierten technischen Fortschritts für den erklärten gesetzmäßigen Sieg des Sozialismus beziehungsweise Kommunismus nachzuweisen hatte, immer stärker zu einem Feld ideologischer Indoktrination und marxistischer Scholastik. Geschuldet war dies seit den 1970er Jahren vornehmlich vom Westen ausgehenden technischen Wandlungen, welche die technologische Lücke zwischen den konkurrierenden Systemen deutlich verbreiterten und den viel berufenen technischen Fortschritt 
endgültig vom revolutionären Akteur im Dienst des Sozialismus $\mathrm{zu}$ dessen fremdbestimmter Existenzbedrohung werden ließ. Disziplinhistorische Ansätze unter dem Dach der Wissenschaftsgeschichte konnten hingegen weitaus politik- und ideologieferner verfolgt werden, zumal die Wissenschaftsgeschichte auch kein Bestandteil des für Studierende aller akademischen Studiengänge der DDR seit 1953 obligaten dreijährigen marxistisch-leninistischen Grundlagenstudiums war.

Überlegungen zum Aufbau der Dresdener Institution und der Entwicklung ihres Lehr- und Forschungsprogramms gingen von dem Befund aus, dass die wachsende Wissenschaftsorientierung von Technik im Industriezeitalter weder in der Wissenschaftsnoch in der Technikgeschichte angemessen reflektiert wurde. Im engeren Sinne rekurrierten die Begründungsdiskurse auf die marxistischen Konzepte der Produktivkraft Wissenschaft und der sogenannten Wissenschaftlich-Technischen Revolution (WTR). Diese waren ihrerseits situiert in der marxistischen Spielart des zu den zentralen Geltungsgeschichten der Hochmoderne zählenden technisierten Fortschrittsoptimismus (Langewiesche 1993, Radkau 1990). Konzepte zum Konnex von gesellschaftlichem Fortschreiten und wissenschaftsorientierter Technik firmierten im real existierenden Sozialismus vornehmlich unter dem Label WTR, einem Dogma an dem die SED-Spitze starr festhielt (Laitko 1996). Dieses Denkmodell hatte seine inhaltliche Ausformung in den 1950er und 1960er Jahren erfahren. Es wies hinsichtlich der szientistischen Leitvorstellung, dass sowohl zwischen technischen und wissenschaftlichen Fortschritten als auch zwischen Technik-, Wirtschaftsund Gesellschaftsentwicklung ein sehr unmittelbarer und vor allem auch planbarer Zusammenhang besteht, wiederum deutliche Parallelen zum sogenannten linearen Modell auf. Letzteres besaß während der ersten Nachkriegsjahrzehnte in den Staaten des Westens handlungsorientierende Kraft in der Wissenschaftsund Technikpolitik (Wengenroth 2008: $61 \mathrm{ff}$.). Doch ist dies nicht die einzige Parallele: Auch im Westen war der historischen Wissenschaftsforschung in der Zeit der Systemkonkurrenz, die ebenso als Wahrheitskonkurrenz gelesen werden kann, eine politische Agenda eingeschrieben (Borck 2009: 317). Während sie hier nach Antworten auf die Frage suchte, wie die Leistungskonkurrenz des Kalten Krieges zu gewinnen sei, sollte das Konstrukt WTR die auf der Dialektik von Produktionsverhältnissen und Produktivkräften gegründete „gesetzmäßige“ Überlegenheit des real existierenden Sozialismus als vermeintlich höhere Gesellschaftsordnung in der 
Wahrheitskonkurrenz nachweisen und damit dessen Herrschaft legitimieren. Im Westen wurde das lineare Modell spätestens im Zuge der Umbrüche in den 1970er Jahren (Doering-Manteuffel/ Raphael 2008) obsolet und fand kaum noch Beachtung in den Diskursen über die sogenannte Zweite (oder Dritte) Industrielle Revolution. Dagegen unterlag das Denkmodell WTR einschließlich des ihm inhärenten Szientismus, obgleich rhetorisch bald von der SED-Führung auf den Index gesetzt und zum „Wissenschaftlich-Technischen Fortschritt" abgerüstet, zwar auch semantischen Metamorphosen und erlitt schließlich einen signifikanten Verlust an Bindekraft, blieb aber bis zum Zusammenbruch der DDR eine zentrale diskursive Leitkategorie der Herrschaftslegitimation und sinnweltlichen Absicherung (Sabrow 2004, Laitko 1996, Radkau 2008: 387 ff., Augustine 2007: 111 ff., 201 ff.). Es bildete zudem in weiten Teilen der Bevölkerung selbst dann noch ein Residuum der Fortschrittshoffnung, nachdem der Glaube an den verheißenen großen Menschheitsfortschritt sich pulverisiert hatte.

Unter Berufung auf diese Begründungszusammenhänge gelang es, das zuständige Ministerium für Hoch- und Fachschulwesen in den 1970er Jahren zu bewegen, monierten Defiziten wissenschaftshistorischer Lehre und Forschung mit einer Förderung dieses Bereichs zu begegnen. Der Arbeitskreis Wissenschaftsgeschichte, 1986 zum Beirat für Wissenschaftsgeschichte aufgewertet, spielte als maßgebender wissenschaftshistorischer Fachvertreter dabei eine wesentliche Rolle, indem es dieses Ministerium beriet. Nun hat in Diktaturen jede Wissenschaft auch die Funktion der Herrschaftslegitimation. Andererseits bilden Wissenschaft und Staat in Diktaturen bekanntlich symbiotische Ermöglichungsverhältnisse zu beiderseitigem Nutzen (Ash 1995: 13 ff.). Daher ließen sich staatliche Ressourcen zur Verfolgung wissenschaftlicher Projekte gleichwohl auch dann rekrutieren, wenn diese zwar nicht primär auf ideologische Indoktrination und klassenkämpferische Propaganda zielten, aber immerhin eine Korrespondenz zum Raster sinnweltlicher Stabilisierung von Herrschaft aufwiesen. Insofern geht die allzu schlichte Vorstellung fehl, in der DDR seien sowohl die Eröffnung neuer wissenschaftlicher als auch das Perpetuieren traditioneller Pfade "von oben“ verordnet worden. Dies setzt die Akteure der Machtblöcke von SED und Ministerialbürokratie ex post in eben jenen Status intellektueller Omnipotenz ein, den sie zu Zeiten des real existierenden Sozialismus reklamiert hatten. Tatsächlich aber waren weder sie noch die politische Elite in demokratisch verfassten 
Staaten in der Lage, fachwissenschaftliche Schwerpunkte vorzugeben. Vielmehr erfolgte dies zunächst über Aushandlungsprozesse auf der Ebene der wissenschaftlichen Akteure. Über diverse Expertengremien traten diese dann den Weg „nach oben“ an, um schließlich als staatliche Vorgabe wieder den Weg in die Niederungen des Wissenschaftsbetriebs zu finden. Damit war auch eine spezifische Form der Akkumulation symbolischen Kapitals verbunden. Letzteres kann, grob vereinfacht, vielleicht mit der Bewilligung von Förderanträgen in der Bundesrepublik verglichen werden.

Daher sind die von Weber/Engelskirchen (2000: 327) in ihrer ein Idealbild West mit einer Karikatur Ost kontrastierenden Geschichte der Technikgeschichte in beiden deutschen Staaten konstruierten Entstehungskontexte der Technikwissenschaftsgeschichte in der DDR empirisch nicht belastbar: Diese sei mit dem Ziel angetreten, die Ingenieurwissenschaften aufzuwerten, womit man zudem auf frühere Bemühungen in der Bundesrepublik reagiert habe. Daneben hätte sie noch ökologische Schäden verschleiern sollen. Weder ließen sich während der 1970er Jahre in der DDR noch ökologische Schäden verbergen (Stoltzfus 2003) was mit akademischer Rhetorik vermutlich ohnehin ins Leere gelaufen wäre - noch hatten Ingenieure und Ingenieurwissenschaften in der DDR im Vergleich zur Bundesrepublik (Rusinek 1996: 299 f., Lenk 1992: 12) eine Aufwertung überhaupt nötig (Zachmann 2006: 296 ff.).

Im Zentrum des Dresdener Konzepts stand die Genese technikwissenschaftlicher Disziplinen. (Buchheim 1980, Guntau/Laitko 1987, Hänseroth/Mauersberger 1996). Es folgte damit der seinerzeit in West und Ost gleichermaßen vollzogenen Ablösung der Ideen-, Begriffs- und Theoriegeschichte durch sozialhistorisch orientierte disziplinhistorische Ansätze. Disziplinen wurden als ein gegenstandsorientiertes System wissenschaftlicher Tätigkeit modelliert, das über längerfristig stabil segmentierte Wissenstraditionen einen einheitlichen Forschungszusammenhang etabliert hat. Es umfasst die Produktion und Reproduktion von Wissen als sozialem Prozess und erhält über die Definition von Rändern zu fremddisziplinären und außerwissenschaftlichen Milieus seine Identität. In diesem Sinne wurde wissenschaftlichen Disziplinen die Funktion eines sozialen Organisations- und kognitiven Orientierungsrahmens einschließlich der Akkreditierung epistemischer Absicherungen zugewiesen. Zentrale Bestimmungsstücke für Disziplingenesen waren gegenständliche Spezifik und Erkenntnisorientierung als Bildungsprinzip sowie die Etablierung eines sowohl wissens- als auch 
akteursbezogenen Reproduktionszusammenhangs bis hin zur Institutionalisierung. Ziel war, auf der Basis vergleichender Studien zu technikwissenschaftlichen Disziplinen nach verallgemeinerungsfähigen Strukturmerkmalen und Bildungsprinzipien vornehmlich in der Phase der Herausbildung der Technikwissenschaften zu suchen. Der zeitliche Schwerpunkt lag insofern auf der Entwicklung der klassischen Technikwissenschaften zwischen dem ausgehenden 17. und frühen 20. Jahrhundert.

Wesentliche Anregungen bezogen die am Zentrum für Geschichte der Technikwissenschaften in Dresden arbeitenden Historiker und Historikerinnen anfangs aus besonders von Buchheim unterhaltenen Kontakten mit sowjetischen Kollegen an den Akademieinstituten in Leningrad (St. Petersburg) und Moskau. Der abgedruckte Aufsatz dokumentiert dies und verweist auf systematische und historische Aspekte, um die zu Beginn der Dresdener Arbeiten die Debatten kreisten. Buchheim, die bis 1955 an der Technischen Hochschule Dresden Berufspädagogik studiert hatte, startete ihre akademische Karriere am dort seit 1952 bestehenden Institut für Geschichte der Technik und Naturwissenschaften. In dessen von politischen Querelen und ideologischen Grabenkämpfen geprägter Auflösungsphase entschied sie sich 1960 als Aspirantin an das Institut für Geschichte der Naturwissenschaft und Technik der Akademie der Wissenschaften der UdSSR nach Moskau zu gehen, wo sie 1965 bei Bonifati Michailowitsch Kedrow mit einer Arbeit über die Entwicklung der Wissenschaft zur unmittelbaren Produktivkraft promoviert wurde. Im gleichen Jahr kehrte sie nach Dresden zurück. Nach anfänglich eher generellen Studien zum Verhältnis von Wissenschaft und Technik verfolgte sie zunehmend einen wissenschaftshistorischen Ansatz, mit dem sie noch am Leningrader Akademieinstitut in Berührung gekommen war. Dort hatten Philosophen und Historiker mit bemerkenswerter zeitlicher Parallelität zu analogen Ansätzen in den USA (Layton 1971, 1974, Staudenmaier 1985: 86 ff., Wengenroth 2003: 244 f.) begonnen, aus systematischer und historischer Perspektive dezidiert die Technikwissenschaften in den Fokus zu nehmen. Diese begriffen sie nicht als lediglich aus den Naturwissenschaften abgeleiteten, sondern als von diesen zu unterscheidenden eigenständigen Typ der noch prinzipiell als Kollektivsingular gedachten Wissenschaft. Mit diesem Ansatz, der ab 1969 von einer um Juri S. Meleschtschenko aufgebauten Gruppe verfolgt wurde, war ein doppelter Paradigmenwechsel vollzogen. Er sollte auch handlungsleitende Kraft für die Entwicklung des Dresdener Konzepts gewinnen: 
Erstens kultivierten zu dieser Zeit Naturwissenschaftler und Naturwissenschaftshistoriker im Westen wie im Osten noch überwiegend das auf die antike Vorstellungswelt eines Natur nachahmenden technischen Handelns verweisende Denkmodell von Technik als angewandter Naturwissenschaft, setzten dies als diskursive Strategie zur Legitimationsbeschaffung ein und leiteten daraus Ansprüche auch auf eine technikhistorische Deutungsmacht ab. Die dann mit dem Dresdener Ansatz in Fallstudien immer wieder herausgearbeitete Eigenständigkeit der Technikwissenschaften fand ihre Begründung vornehmlich in dem empirisch dicht belegten Befund, dass die Produktion technikwissenschaftlichen Wissens, wenn auch mit stark oszillierenden Schwerpunktsetzungen, stets an sowohl die Wissenschaft als auch die technische Praxis adressiert war. Dagegen vermochte naturwissenschaftliches Wissen überwiegend nur über einen Reaktionsraum, eben die Technikwissenschaften, in technische Anwendungskontexte zu gelangen. Gleichwohl wurde bewusst gehalten, dass naturwissenschaftliches Wissen auch auf direktem Wege technisches Handeln orientieren kann. Vice versa wurde dieser Reaktionsraum ebenso als Vermittlungsinstanz von Anforderungen aus der technischen Praxis an naturwissenschaftliche Forschung begriffen. Mit zunehmender Ausprägung ihrer Eigenständigkeit, spezifischen Funktion und epistemischen Qualität, so das Denkmodell, würden Technikwissenschaften dieser doppelten Vermittlung immer besser genügen.

Zweitens ging das Dresdener Konzept davon aus, dass ungeachtet unscharfer Ränder wissenschaftliches und technisches Handeln jeweils spezifischen Zielen folge und insofern selbst umstürzende wissenschaftliche Ergebnisse noch lange nicht patentfähig seien. Zudem besteht zwischen epistemischen und technischen Dingen keine bloß semantische Differenz, sondern erfolgreiches technisches Handeln ist auch in Zeiten seiner zunehmenden Wissenschaftsorientierung in einem Kontinuum von Wissen und Können sehr unterschiedlicher Provenienz situiert (Hänseroth/Mauersberger 1998, Banse u. a. 2006, Wengenroth 2008: 71 ff.). Methodische Prämisse war insofern nicht nur die Abgrenzung von den Naturwissenschaften. Dies galt gleichermaßen für die Technik. Technikhistoriker rückten seinerzeit zwar zunehmend von der Vorstellung ab, Technik sei als angewandte Wissenschaft zu fassen und stellten im Bemühen um eine differenziertere Sicht die Verwissenschaftlichung von Technik stärker in den Fokus. Sie schwenkten dabei aber relevantes wissenschaftliches Wissen wie einen Deus ex machina auf die Bühne der Technikgeschichte. Dagegen galt das zentrale 
Erkenntnisinteresse des Dresdener Ansatzes nicht der Verwissenschaftlichung von Technik oder dem Anteil wissenschaftlichen Wissens am Zustandekommen funktionierender Technik, sondern der Entstehung und Ausdifferenzierung wissenschaftlich-technischen Wissens als historisch jüngster Wissensform technischen Handelns und als gruppenspezifisches, identitätsstiftendes Sonderwissen von Ingenieuren. Obwohl Technikwissenschaftshistoriker den permanenten Wandel ihrer Gegenstands- und Objektbereiche durch menschliches Handeln konzeptualisieren mussten und insofern die Technikwissenschaftsgeschichte immer auch technikhistorische Elemente inkorporiert hatte, begriffen sich die Dresdener Akteure eher als Wissenschaftshistoriker. Dies waren zudem gute inhaltliche Gründe für die praktizierte enge Anbindung der Technikwissenschaftsgeschichte an die Interessenvertretungen und Netzwerke der Wissenschaftsgeschichte in der DDR.

Leitfragen des dezidiert wissenschaftshistorisch orientierten Forschungsprogramms galten besonders Problemlagen der Herausbildung, Perpetuierung und Wandlung technikwissenschaftlicher Disziplinen, ihrer Spezifik im Vergleich mit vermeintlichen naturwissenschaftlichen Urbildern (wie etwa der Differenz von Technischer Mechanik und Festkörperphysik), der Gegenstandsorientierung, den Erkenntnissystemen, den Praktiken und Räumen der Produktion, Autorisierung und Normierung von Wissen, den wissenschaftlichen Akteuren, den akademischen und außerakademischen Institutionalisierungsformen, Periodisierungen sowie der Applikationsfähigkeit von Theorieangeboten. Letzteres rückte, freilich aus der Perspektive der Disziplingenese, auch das Verhältnis von Wissenschaft und Technik in den Fokus.

Forschungserträge finden sich besonders in den ab 1980 erschienenen Dresdener Beiträgen zur Geschichte der Technikwissenschaften, der NTM - die als wichtigstes wissenschafts- und technikhistorisches Publikationsorgan der DDR wesentlich für die Ausbreitung der Dresdener Arbeiten und nicht zuletzt auch deren Kenntnisnahme in der Bundesrepublik (Blumtritt 1988, Wengenroth 2003) sorgte - sowie in zwei Sammelbänden (Buchheim/Sonnemann 1989, 1990). Eine erste Bilanz konnte 1986 auf dem Symposium des International Committee for the History of Technology (ICOHTEC) in Dresden gezogen werden (Sonnemann/Krug 1987). Es hatte erstmals in der Geschichte der ICOHTEC-Tagungen, die in der Zeit des Kalten Krieges Technikhistoriker aus West und Ost zusammenbringen sollten, expressis verbis die Wissens- und Wissenschaftsgeschichte der Technik zum Thema. 
Zu den Aktiva der Dresdener Arbeiten zählt meines Erachtens zunächst, dass unter den Bedingungen von Geschichtswissenschaft in der DDR im Vergleich zum traditionellen und anderenorts bis zum Zusammenbruch perpetuieren Ansatz der Geschichte der Produktivkräfte ein deutlich aussagekräftigeres Konzept zur Fokussierung wissenschaftshistorischer Fragestellungen entwickelt wurde. Damit gelang zugleich die methodische wie institutionelle Emanzipation von der Geschichte der Produktivkräfte. Unter diesen Prämissen konnten im Zusammenspiel von systematischen und historischen Fragestellungen Aufkommen und Etablierung der Technikwissenschaften - besonders mit Blick auf charakteristische Gegenstände, epistemische Dispositionen sowie Institutionalisierungs- und Reproduktionsformen - als eines genuinen Wissenschaftstyps herausgearbeitet und ihre Genese anhand eines Kriterienkatalogs jeweils abgelaufener Prozesse periodisiert werden. Das in Dresden entwickelte Periodisierungsmuster unterschied zwischen der Genese des Wissenschaftstyps generell und einzelnen Disziplingenesen. Es rekurriert auf die Stadien Vorgeschichte, Herausbildung und Konsolidierung (Hänseroth/Mauersberger 1996: 27 ff).

Dagegen weist das Dresdener Konzept blinde Flecken besonders in Bezug auf die kulturelle Situiertheit von Wissenschaft und ein reflexives Wissenschaftsverständnis auf. Gegenüber der dominierenden Analyse von Strukturen und ihren Gesetzmäßigkeiten geriet das Handeln individueller Akteure oft in den Hintergrund. So aber lassen sich zum Beispiel disziplinäre Aushandlungsprozesse, die Wirkmacht kultureller Imprägnierungen oder auch die Bedeutung personengebundenen impliziten Wissens nicht angemessen erfassen. Zudem war das Konzept produzentenfixiert. Vor allem aber leiteten positivistischer Erkenntnisoptimismus und Szientismus die Arbeiten mit dem Dresdener Ansatz. Insofern spiegelt sich auch in den Dresdener Arbeiten die Sklerose der Gesellschaftswissenschaften der DDR wider. Andererseits ließ die wachsendene Anzahl empirischer Befunde aus Fallstudien die dem marxistischem historischen Denken eingeschriebene Vorannahme möglicher Gesetzeserkenntnis sowie deren Anwendung auf gegenwärtige und künftige gesellschaftliche Zustände zunehmend prekär werden. Infolgedessen wurden im Verlauf der 1980er Jahre die Deutungsangebote der Dresdener Technikhistoriker deutlich zurückhaltender formuliert. Die hier sozialisierten Fachvertreter bauten dabei eine Sensibilität im Umgang mit empirischen Befunden auf und haben sich wohl auch späterhin Skepsis gegenüber dem allzu reichlichen Abbrennen großtheoretischer Pyrotechnik bewahrt. 
Ein Desiderat aus zeitlicher Perspektive blieb die als moderne Periode bezeichnete Phase ab etwa 1950. Dies gilt besonders hinsichtlich der systematischen Auswertung einiger immerhin entstandener Fallstudien. So gerieten auch die sich jetzt einstellenden engen Kopplungen von Wissenschaft und Technik (Weingart 2001) oder auch deren postuliertes Verschmelzen zur Technoscience (Latour 1987) nicht zum Gegenstand systematischer Überlegungen. Ex post mag diese zeitliche Beschränkung angesichts der oben genannten Legitimationskontexte der Geschichte der Technikwissenschaften in der DDR, die nicht zuletzt auf die ,gegenwärtige WTR“ rekurrierten, nachgerade surreal anmuten. Vielleicht aber scheute schlicht die Mehrzahl der Technikwissenschaftshistoriker allzu große zeitliche Nähe zur real existierenden DDR, um erwarteter Gesellschafts- und Systemapologetik zu entgehen.

Die Arbeiten mit dem Dresdener Ansatz brachen Anfang der 1990er Jahre weitgehend ab. Zunächst war dies dem Umstand geschuldet, dass, disziplinhistorisch betrachtet, wesentliche Institutionalisierungs- und Reproduktionszusammenhänge nach der deutschen Wiedervereinigung in den neuen Bundesländern nicht mehr gegeben waren. Darüber hinaus aber hatten sich für einige Akteure der Dresdener Gruppe, zu denen auch der Autor dieses Kommentars zählt, die Potenzen des disziplinhistorischen Konzepts inzwischen erschöpft und spannende wissenschaftshistorische Fragen sich jenseits disziplinärer Ordnungen ergeben.

\section{Literatur}

Ash, Mitchel G., 1995. Wissenschaftswandel in Zeiten politischer Umwälzungen: Entwicklungen, Verwicklungen, Abwicklungen. NTM. Zeitschrift für Geschichte und Ethik der Naturwissenschaften, Technik und Medizin, 3, 1-21.

Augustine, Dolores L., 2007. Red Prometheus. Engineering and Dictatorship in East Germany, 1945-1990. Cambridge, Mass. u. a.: MIT Press.

Banse, Gerhard u. a., Hg., 2006. Erkennen und Gestalten. Eine Theorie der Technikwissenschaften. Berlin: edition sigma.

Blumtritt, Oskar, 1988. Genese der Technikwissenschaften. Ein Resümee methodologischer Konzepte. Technikgeschichte, 55, 75-86.

Borck, Cornelius, 2009. Wozu historische Wissenschaftsforschung? Berichte zur Wissenschaftsgeschichte, 32, 315-318.

Buchheim, Gisela, 1980. Zur Herausbildung der Technikwissenschaften. Probleme wissenschaftshistorischer Untersuchungen. Dresdener Beiträge zur Geschichte der Technikwissenschaften, 1, 12-34.

Buchheim, Gisela/Sonnemann, Rolf, 1985. Zur Geschichte der Technikwissenschaften in der DDR. NTM. Schriftenreihe für Geschichte der Naturwissenschaften, Technik und Medizin, 22, 19-24. 
Buchheim, Gisela/Sonnemann, Rolf, 1986. Die Geschichte der Technikwissenschaften - eine neue wissenschaftshistorische Disziplin. Wissenschaftliche Zeitschrift der TU Dresden, 35, 135-142.

Buchheim, Gisela und Sonnemann, Rolf, Hg., 1989. Lebensbilder von Ingenieurwissenschaftlern. Leipzig: Edition.

Buchheim, Gisela und Sonnemann, Rolf, Hg., 1990. Geschichte der Technikwissenschaften. Leipzig: Edition und Basel u. a.: Birkhäuser.

Doering-Manteuffel, Anselm/Raphael, Lutz, 2008. Nach dem Boom. Perspektiven auf die Zeitgeschichte seit 1970, Göttingen: Vandenhoeck \& Ruprecht.

Guntau, Martin/Laitko, Hubert, 1987. Entstehung und Wesen wissenschaftlicher Disziplinen. In: Martin Guntau und Hubert Laitko, Hg., Der Ursprung der modernen Wissenschaften: Studien zur Entstehung wissenschaftlicher Disziplinen. Berlin (Ost): Akademie-Verlag, 17-89.

Hänseroth, Thomas/Mauersberger, Klaus, 1996. Das Dresdener Konzept zur Genese technikwissenschaftlicher Disziplinen - eine Bilanz. Dresdener Beiträge zur Geschichte der Technikwissenschaften, 24, 20-45.

Hänseroth, Thomas/Mauersberger, Klaus, 1998. Technikwissenschaften zwischen theoretischer Erkenntnis und Ingenieurtätigkeit. NTM. Internationale Zeitschrift für Geschichte und Ethik der Naturwissenschaften, Technik und Medizin, 6, 217-237.

Laitko, Hubert, 1989. Geschichte der Technikwissenschaften. Ihr Eigenwert und ihre Bedeutung für die allgemeine Geschichte der Wissenschaft. Dresdener Beiträge zur Geschichte der Technikwissenschaften, 18, 3-47.

Laitko, Hubert, 1996. Wissenschaftlich-technische Revolution. Akzente des Konzepts in Wissenschaft und Ideologie der DDR. Utopie kreativ, 73/74, 33-50.

Langewiesche, Dieter, 1993. Fortschritt als sozialistische Hoffnung. In: Klaus Schönhoven und Dieter Staritz, Hg., Sozialismus und Kommunismus im Wandel: Hermann Weber zum 65. Geburtstag. Köln: Bund-Verlag, 39-55.

Latour, Bruno, 1987. Science in Action. How to Follows Scientists and Engineers Through Society. Cambridge, Mass. u. a.: MIT Press.

Layton, Edwin T. Jr., 1971. Mirror-Image Twins: The Communities of Science and Technology in 19th-Century America. Technology and Culture, 12, 562-580.

Layton, Edwin T. Jr., 1974. Technology as Knowledge. Technology and Culture 15, 31-41.

Lenk, Hans, 1992. Technische Intelligenz im Spannungsfeld von Wissenschaft, Technik, Gesellschaft und Politik. In: Else Fricke, Hg., Interdisziplinäre Technikforschung und Ingenieurausbildung, Bonn: Friedrich-Ebert-Stiftung, 7-18.

Radkau, Joachim, 1990. Revoltierten die Produktivkräfte gegen den real existierenden Sozialismus? Technikhistorische Anmerkungen zum Zerfall der DDR. 1999. Zeitschrift für Sozialgeschichte des 20. und 21. Jahrhunderts, 5, 13-42.

Radkau, Joachim, 2008. Technik in Deutschland. Vom 18. Jahrhundert bis zur Gegenwart. Frankfurt a. M.: Campus.

Rusinek, Bernd A., 1996. Kernenergie, Kernforschung und „Geschichte”: Zur historischen Selbst- und Fremdeinordnung einer Leitwissenschaft. In: Burkhard Dietz u. a., Hg., Technische Intelligenz und „Kulturfaktor Technik”. Kulturvorstellungen von Technikern und Ingenieuren zwischen Kaiserreich und früher Bundesrepublik Deutschland. Münster u. a.: Waxmann, 297-316.

Sabrow, Martin, 2004. Zukunftspathos als Legitimationsressource. Zu Charakter und Wandel des Fortschrittparadigmas in der DDR. In: Heinz-Gerhard Haupt und Jörg Requate, Hg., Aufbruch in die Zukunft. Die 1960er Jahre zwischen Planungseuphorie und kulturellem Wandel. DDR, CSSR und Bundesrepublik Deutschland im Vergleich. Weilerswist: Velbrück Wissenschaft, 165-184.

Sonnemann, Rolf/Krug, Klaus, Hg., 1987. Technology and Technical Sciences in History. Proceedings of the ICOHTEC-Symposium 1986. Berlin (Ost): VEB Deutscher Verlag der Wissenschaften.

Staudenmaier, John M., 1985. Technology's Storytellers. Reweaving the Human Fabric. Cambridge, Mass. u. a.: MIT Press. 
Stoltzfus, Nathan, 2003: Public Space and the Dynamics of Environmental Action: Green Protest in the German Democratic Republic. Archiv für Sozialgeschichte, 43, 385-403.

Weber, Wolfhard/Engelskirchen, Lutz, 2000. Streit um die Technikgeschichte in Deutschland 1945-1975. Münster: Waxmann.

Weingart, Peter, 2001. Die Stunde der Wahrheit? Zum Verhältnis der Wissenschaft zu Politik, Wirtschaft und Medien in der Wissensgesellschaft. Weilerswist: Velbrück Wissenschaft.

Wengenroth, Ulrich, 2003. Science, Technology, and Industry. In: David Cahan, Hg., From Natural Philosophy to the Sciences. Writing the History of Nineteenth-Century. Chicago u. a.: University of Chicago Press, 221-253.

Wengenroth, Ulrich, 2008. Innovationspolitik und Innovationsforschung. In: Gerd Graßhoff und Rainer C. Schwinges, Hg., Innovationskultur. Von der Wissenschaft zum Produkt. Zürich: vdf Hochschulverlag, 61-77.

Zachmann, Karin, 2006. Vom Industrie- zum Staatsangestellten: Die Ingenieure in der SBZ/DDR 1945-1989. In: Walter Kaiser und Wolfgang König, Hg., Geschichte des Ingenieurs. Ein Beruf in sechs Jahrtausenden. München: Hanser, 269-307.

Thomas Hänseroth

Lehrstuhl für Technik- und Technikwissenschaftsgeschichte

Philosophische Fakultät

TU Dresden

01062 Dresden

Deutschland

E-Mail: Thomas.Haenseroth@tu-dresden.de 